\title{
Entre Mexique et États-Unis : la chaîne entrepreneuriale de la mort des migrants
}

Between Mexico and the United States: The Entrepreneurial Chain of Migrants' Death

Entre México y Estados-Unidos: la cadena empresarial de la muerte de los migrantes

\section{Françoise Lestage}

\section{OpenEdition}

Journals

Édition électronique

URL : https://journals.openedition.org/remi/6049

DOI : $10.4000 /$ remi.6049

ISSN : $1777-5418$

Éditeur

Université de Poitiers

Édition imprimée

Date de publication : 1 septembre 2012

Pagination : 71-88

ISBN : 979-10-90426-05-4

ISSN : 0765-0752

\section{Référence électronique}

Françoise Lestage, «Entre Mexique et États-Unis : la chaîne entrepreneuriale de la mort des

migrants ", Revue européenne des migrations internationales [En ligne], vol. 28 - n³ | 2012, mis en ligne le 01 septembre 2015, consulté le 15 avril 2022. URL : http://journals.openedition.org/remi/6049 DOI : https://doi.org/10.4000/remi.6049 


\section{Entre Mexique et États-Unis : la chaîne entrepreneuriale de la mort des migrants}

\section{Françoise Lestage ${ }^{1}$}

\section{Introduction ${ }^{2}$}

Depuis la fin du XIXe siècle, la conjoncture économique et politique du Mexique et des États-Unis impulse des politiques migratoires changeantes et des mouvements incessants de personnes entre les deux pays. Attirés sur les grands chantiers étatsuniens à la fin du XIXe et au début du XXe siècles en remplacement des migrants chinois, puis dans des programmes agricoles ou industriels de 1942 à $1964^{3}$, les Mexicains ont bénéficié des régularisations massives de 1986 avant de subir les effets de la fermeture des frontières étatsuniennes post-septembre 2001 et ceux de la crise économique de la fin des années 2000. Pendant près d'un siècle, la majorité des migrants provenait des États du centre et du centre-nord du Mexique (Michoacán, Guanajuato, Jalisco, Zacatecas) ; depuis les années 1980, les migrants d'autres États ont rejoint massivement ce flux (Oaxaca, Guerrero). Tout au long du siècle passé, les évènements politiques et économiques ont donc entraîné des vagues migratoires successives, saisonnières et pendulaires, mais aussi des installations aux États-Unis, des retours au Mexique et enfin des familles qui résident dans les deux pays, une situation propice à des va-et-vient plus ou moins fréquents.

Les transferts des dépouilles des migrants mexicains s'inscrivent dans cette longue histoire. Tout comme les vivants, mais en sens inverse, les corps des Mexicains décédés aux États-Unis empruntent, selon les époques et les circonstances, des routes terrestres, ferrées ou aériennes. Dans les années 2000, le transfert des corps au pays d'origine se fait soit par terre, avec des entreprises de pompes funèbres étatsuniennes puis mexicaines, soit par avion. À l'arrivée dans un aéroport mexicain, le cercueil est pris en charge par des entreprises de pompes funèbres locales qui l'acheminent jusqu'à la maison du défunt. Ces entreprises constituent des acteurs pivots qui organisent, accompagnent et facilitent les transferts des restes humains. Certaines d'entre elles se sont spécialisées dans ce que l'on peut qualifier de véritable business, réglant les questions pratiques et administratives du

1 Professeur d'anthropologie, Université Paris Diderot, Laboratoire URMIS (UMR 205), case courrier 7027, 75205 Paris cedex 13 ; francoise.lestage@univ-paris-diderot.fr

2 Je remercie Gail Mummert et Guillermo Fernandez, chercheurs à El Colegio de Michoacán

(Zamora, Mexique), qui m'ont mise en contact avec les responsables de l'association de

Tangancícuaro. Je remercie également, à Tangancícuaro, don Roman Oropesa Reyes et Jorge

Gudino Rios ; à Oaxaca, Heladio Ramirez; et à Tijuana, Sergio Valencia. Je remercie enfin Rufino

Dominguez, directeur de I'IOAM depuis décembre 2010, qui a facilité mes recherches.

3 Programme dit Bracero. 
voyage du cercueil et, parfois, des proches qui accompagnent le défunt; le passage légal d'un pays à l'autre constitue en quelque sorte leur fonds de commerce.

Dans cet article, je m'intéresse à la place qu'occupe cette industrie mexicaine du transfert funéraire international dans le processus migratoire en $\mathrm{m}^{\prime}$ appuyant sur l'ethnographie de trois entreprises spécialisées dans cette niche commerciale. II s'agit d'interroger les liens qu'elles entretiennent avec d'autres acteurs des transferts des corps que sont l'État fédéral et les États régionaux au Mexique, les familles des défunts, les associations de migrants et d'autres entreprises de pompes funèbres au Mexique et aux États-Unis.

\section{Des entreprises de pompes funèbres destinées aux migrants}

Les trois entreprises mexicaines concernées par l'enquête sont installées dans trois régions différentes, de 1000 à 3000 kms de distance, aux caractéristiques migratoires distinctes : une entreprise familiale dans le sud du pays (Oaxaca, État du Oaxaca) ; un auto-entrepreneur dans le nord du pays (Tijuana, État de Basse-Californie) ; une entreprise appartenant à une association mutualiste dans le centre du pays (Tangancícuaro, État du Michoacán). Pour celles établies à Tangancícuaro et à Oaxaca, leur travail consiste à transférer des migrants défunts des États-Unis vers le Mexique. Par contre pour celle de Tijuana, les transferts sont aussi nombreux du Mexique vers les États-Unis que des États-Unis vers le Mexique ; la situation frontalière induisant des spécificités sur lesquelles je reviendrai. II convient cependant de souligner que les transferts des corps d'un pays à l'autre ne représentent qu'une partie des services fournis par ces entreprises qui proposent également la mise à disposition d'une salle pour les veillées funéraires, la location de "kits-veillée " (objets nécessaires pour une veillée funéraire à domicile), ou encore une aide administrative et linguistique aux familles (cf. tableau 1). Excepté I'autoentrepreneur deTijuana, dont la tâche consiste à se rendre d'un territoire national à l'autre pour faire passer la frontière aux défunts, les deux autres entreprises exercent leur activité dans les limites territoriales de l'État mexicain que leurs responsables n'ont jamais quitté pour travailler aux États-Unis, ce qui n'est pas si courant dans un pays où un séjour de travail dans le pays voisin est banal et fréquent.

\section{Une entreprise familiale de pompes funèbres (Oaxaca, Oaxaca)}

Dans l'État du Oaxaca, deuxième État fédéré "expulseur " de migrants au Mexique ${ }^{5}$, où la migration vers les États-Unis s'est généralisée au cours des années 1980, I'Institut national d'Aide au Migrant (IOAM) de l'État fédéré prend en charge l'organisation et le financement d'une partie du transfert : le transport de l'aéroport mexicain à la maison du défunt, y compris dans les localités les plus reculées. De 2000 à 2010, I'IOAM a confié cette tâche à une seule entreprise de pompes funèbres qui en a ainsi obtenu le monopole.

Entreprise familiale ${ }^{6}$ fondée en 2000 dans la ville d'Oaxaca (300 000 habitants), capitale de l'État du même nom, par un retraité de l'hôpital, Heladio, employé au service

4 " Kit " composé de deux chandeliers, deux vases, un rideau pour attacher le crucifix et un trépied pour poser le cercueil. Le terme de " kit » est celui qu'utilise Heladio, propriétaire de l'entreprise La Purísima à Oaxaca (entretiens juin 2007, avril 2008 et mars 2010).

5 Le premier est I'État du Michoacán. Cf. INEGI (2010) Censo de población y vivienda.

6 II s'agit d'une entreprise familiale ayant deux établissements : I'un dans la ville d'Oaxaca, tenu par le propriétaire, son fils et son gendre ; l'autre dans une petite agglomération à une dizaine de kilomètres d'Oaxaca tenu par sa fille et son épouse. 
Tableau 1 : Caractéristiques des trois entreprises de pompes funèbres étudiées

\begin{tabular}{|l|l|l|l|}
\hline Caractéristiques & \multicolumn{1}{|c|}{$\begin{array}{c}\text { Entreprise PF } \\
\text { de I'association } \\
\text { mutualiste } \\
\text { Tangancícuaro }\end{array}$} & \multicolumn{1}{|c|}{$\begin{array}{c}\text { Entreprise PF } \\
\text { La Purísima }\end{array}$} & $\begin{array}{c}\text { Auto-entrepreneur } \\
\text { Sergio Valencia }\end{array}$ \\
\hline Type juridique & associatif & $\begin{array}{l}\text { entreprise } \\
\text { commerciale }\end{array}$ & auto-entreprise \\
\hline Localisation & $\begin{array}{l}\text { Tangancícuaro, } \\
\text { Michoacán, Mexique }\end{array}$ & $\begin{array}{l}\text { Santa María del Tule et } \\
\text { Oaxaca, Mexique }\end{array}$ & $\begin{array}{l}\text { Tijuana, Basse } \\
\text { Californie, Mexique }\end{array}$ \\
\hline Début d'activité & $\begin{array}{l}1984 \\
\text { (association : 1966) }\end{array}$ & 1998 & 1983 \\
\hline Local spécifique & oui & oui & non \\
\hline Employés permanents & $\begin{array}{l}1 \\
\text { (+2 dans I'association) }\end{array}$ & 3 & 1 \\
\hline $\begin{array}{l}\text { Moyenne de transferts } \\
\text { US vers MX par an }\end{array}$ & 20 & 200 & 150 à 250 \\
\hline Transferts MX vers US & occasionnel & occasionnel & 150 à 250 \\
\hline $\begin{array}{l}\text { Transport des cadavres } \\
\text { en corbillard }\end{array}$ & $\begin{array}{l}\text { oui } \\
\text { (1 seul à la fois) }\end{array}$ & $\begin{array}{l}\text { oui } \\
\text { (1 seul à la fois) }\end{array}$ & $\begin{array}{l}\text { oui } \\
\text { (1 à } 6 \text { à la fois) }\end{array}$ \\
\hline Vente de cercueils & oui & oui & non \\
\hline $\begin{array}{l}\text { Location de kit veillée } \\
\text { (chandeliers, trépied, } \\
\text { vases, crucifix) }\end{array}$ & oui & oui & non \\
\hline $\begin{array}{l}\text { Location de salle pour } \\
\text { veillée funèbre }\end{array}$ & oui & non & non \\
\hline $\begin{array}{l}\text { Gestion des démarches } \\
\text { de rapatriement des } \\
\text { corps }\end{array}$ & oui & $\begin{array}{l}\text { assurance décès } \\
\text { et invalidité pour } \\
\text { migrants (US) } \\
\text { et non-migrants } \\
\text { (MX) proposés par } \\
\text { au passage de la } \\
\text { frontière terrestre. }\end{array}$ & nomilles des défunts \\
\hline nutres services & eutatsuniens à Tijuana \\
\hline
\end{tabular}

Légende : US = États-Unis ; MX = Mexique ; les villes sont mentionnées en premier et suivies du nom de l'État régional (Tangancícuaro, Michoacán). 
funéraire jusqu'en 1998, I'entreprise La Purísima a été progressivement impliquée dans les transferts des migrants défunts jusqu'à en faire la plus grande partie de son activité commerciale. En 2007, elle avait pris en charge environ 300 décès, dont 247 transferts financés, totalement ou partiellement, par I'IOAM. À partir de 2008, la suppression des grands avions sur la ligne de Mexico à Oaxaca a marqué la fin du transport aérien des cercueils jusqu'à Oaxaca. C'est donc à partir de l'aéroport de Mexico, à six heures de route, que s'effectue dorénavant le transport terrestre. Dès lors, l'entreprise a limité ses transports aux défunts arrivant directement deTijuana. Pourtant, grâce à la relation privilégiée que son propriétaire entretient avec une des responsables du service dédié aux transferts des défunts de I'IOAM, elle a conservé malgré tout le monopole du transfert local en sous-traitant avec une entreprise de pompes funèbres de Mexico (à $300 \mathrm{kms}$ environ) et a continué de coordonner les transferts avec les entreprises étatsuniennes et avec les familles aux États-Unis et au Mexique. En décembre 2010, un changement de gouverneur ${ }^{7}$ et de parti au pouvoir dans l'État de l'Oaxaca a entraîné la nomination d'un nouveau directeur de I'IOAM qui a cessé sa collaboration avec La Purísima, trop chère selon lui ${ }^{8}$, et a signé un contrat avec deux autres entreprises de pompes funèbres locales qui se partagent le marché depuis janvier 2011.

Au moment où il a créé son entreprise, Heladio avait déjà des compétences dans le commerce de la mort puisqu'il avait été formé par l'hôpital à traiter avec des familles en deuil. Pour lui, son empathie avec ses clients et son inclination à "les consoler " ${ }^{9}$ sont à distinguer des relations marchandes anonymes de la plupart des commerces (alors qu'il $s^{\prime}$ agit là d'une constante dans la rhétorique des entrepreneurs de pompes funèbres $\left.{ }^{10}\right)$. La situation de monopole de La Purísima de 2000 à 2010 lui a permis de bâtir un réseau de relations avec des entreprises de pompes funèbres étatsuniennes avec l'objectif de traiter directement avec elles (alors qu'il ne garde aucune archive, il a pris la peine de noter sur un cahier les noms et adresses des entreprises étatsuniennes avec qui il était en contact) et d'acquérir un autre savoir-faire - celui du transfert international des corps en provenance des États-Unis - ; un savoir-faire et un réseau entrepreneurial qu'il tente désormais de valoriser alors même qu'il ne bénéficie plus du monopole.

\section{Une entreprise de pompes funèbres propriété d'une association mutua- liste (Tangancícuaro, Michoacán)}

Située dans le centre du Mexique, à Tangancícuaro (15 000 habitants) dans l'État du Michoacán, premier État " expulseur " de migrants au Mexique et principal fief des " braceros" (travailleurs légalement embauchés par des entreprises étatsuniennes de 1942 à 1964), l'entreprise de pompes funèbres enquêtée appartient à une association mutualiste fondée par des migrants pour assurer le financement des transferts de corps depuis les États-Unis ${ }^{11}$.

\footnotetext{
7 Le gouverneur de l'État fédéré est élu par les habitants pour quatre ans. II est en quelque sorte le président de l'État fédéré et de lui dépendent toutes les administrations. II nomme les hauts fonctionnaires.

8 Interview Rufino Dominguez, directeur de I'IOAM depuis le 1er décembre 2010 (novembre 2011). 9 Entretien Heladio Ramirez, Oaxaca, 28 mars 2010.

10 Voir Trompette et Boissin, 2000 ; Trompette et Caroly, 2004 ; Bernard, 2008 ; Trompette, 2008. Comme le souligne Bernard (2008), " la capacité et la mise en œuvre d'empathie s'avèrent donc une ressource pour apporter un "supplément d'âme" à la relation, mais elle n'est pas toujours possible ".

11 L'association administre l'entreprise de pompes funèbres et il est impossible de séparer l'une de l'autre, même si l'entreprise peut, en principe, vendre ses services à d'autres clients que les membres de I'association. La raison d'être de l'association reste le financement des obsèques et des transferts de corps.
} 
En 2009, I'Association Mutualiste de Tangancícuaro'12 comptait 2591 membres, dont 1213 femmes et 1378 hommes $^{13}$, résidant dans le municipe ${ }^{14}$ ou aux États-Unis, en particulier en Californie, dans la région d'agriculture industrielle allant de Los Angeles à Sacramento. Propriétaire d'un petit immeuble de deux étages donnant sur la place principale de la localité qui comprend une salle de réception et les bureaux modernes et confortables du gérant et de la secrétaire, tous deux employés à plein temps, I'association a acheté en 1984 une entreprise de pompes funèbres qui réalise une vingtaine d'enterrements par an - vingt en 2009 - soit à la suite d'un transfert de corps depuis les ÉtatsUnis, soit à l'occasion du décès d'un membre résidant sur place. L'entreprise est située à une centaine de mètres du bâtiment de l'association dans un édifice abritant une grande salle destinée à des veillées funèbres, un entrepôt de cercueils à vendre, un logement pour la famille de l'homme à tout faire, gardien de l'entreprise et chauffeur rémunéré par I'association. Celle-ci propose également des assurances à ses membres, notamment une assurance-décès qui sert à couvrir les frais des obsèques et, dans certains cas, ceux du transfert du corps depuis les États-Unis, très exceptionnellement du Mexique vers les États-Unis. Au premier janvier 2010, les membres payaient, en moyenne, une cotisation de soixante-dix dollars par an alors que l'association mutualiste versait à ses membres 70000 pesos (5 500 dollars) que les familles utilisaient comme elles l'entendaient.

L'association mutualiste fut fondée en 1966 par trois migrants dont seul Roman, premier secrétaire, était encore en vie lors de mon enquête en 2010. Au cours de notre entretien, il m'expliqua sa décision de participer à la création d'une structure pérenne suite à I'humiliation ressentie en 1963 quand il dut solliciter ses compatriotes émigrés en Californie, "comme un mendiant " ${ }^{15}$ à l'occasion du décès de son frère dans un accident de voiture. Roman mit une dizaine de jours à réunir la somme nécessaire au rapatriement du corps, délai qui fut une épreuve pour lui, mais aussi pour ses parents qui vivaient au Mexique dans l'attente du corps. C'est lui qui accompagna le cercueil dans un voyage en train long d'une semaine. Dans les années qui suivirent, avec d'autres migrants travaillant dans la même entreprise, il œuvra à la mise en place de l'association en utilisant le modèle d'une association mutualiste qui, selon ses dires, fonctionnait déjà à Tijuana (Basse-Californie, Mexique) à l'époque et dont lui et ses camarades obtinrent une copie des statuts.

L'association mutualiste s'est transformée à la suite d'évènements successifs : au début des années 1970, elle a acquis un local, ce qui lui a donné une plus grande visibilité ; au milieu des années 1970, après d'âpres discussions, les non-migrants de la localité ont été autorisés à entrer dans l'association où ils sont devenus majoritaires dès 1980, ce qui a posé la question des intérêts des uns et des autres, souvent divergents ; en janvier 1983, I'association a racheté une entreprise de pompes funèbres, ouverte à tous, qui pratique les mêmes tarifs que les entreprises locales; à la fin des années 1980, I'association a créé une agence de voyages qui n'a que peu fonctionné, faute de clients. Bien que le gérant dise qu'il s'agit " d'investir et de placer l'argent pour qu'il rapporte et pas de faire du

12 La population totale du municipe était en 2010 de 32677 habitants dont 30548 sont déclarés nés dans le municipe et seulement 297 vivaient dans un autre lieu lors du recensement de 2005 et sont donc considérés comme migrants. La localité même de Tangancicuaro (hors villages environnants) comptait dans le même recensement 15068 habitants. Cf. INEGI (2012) Censo de población y vivienda.

13 Gráfica de número de socios, 2009 (Graphique du nombre de membres de l'association), document interne.

14 Un municipe est une division administrative qui comprend un bourg principal et les localités environnantes.

15 Roman Reyes Oropesa, né en 1936 au Mexique, reconnu comme immigré légal aux États-Unis en 1958 (interview du 3 mars 2010). 
profit $\|^{16}$, l'association mutualiste s'est comportée comme une petite entreprise, spécialisée dans le commerce de la mort : elle achète des immeubles et des terrains et investit dans des affaires plus ou moins liées à son objectif original, celui de prendre en charge financièrement et matériellement le retour des défunts dans leur région d'origine. Depuis 2010, I'association indemnise aussi ses adhérents, pour la plupart travailleurs agricoles, en cas de perte de l'usage d'un membre.

Depuis sa création, elle a donc connu deux évolutions majeures : une moindre utilisation comme aide au rapatriement des défunts et une fonction accrue comme assureur local. En effet, alors qu'elle était constituée exclusivement de migrants travaillant ensemble aux États-Unis, elle a élargi ses services à des Philippins, Portoricains et Chiliens $^{17}$, puis s'est progressivement tournée vers les habitants des localités d'origine comme on le constate dans les documents récents : en 2010 les présidents, secrétaires et trésoriers résident au Mexique alors qu'à la création de l'association, ils vivaient en Californie ; en 2009, les versements destinés aux familles des mutualistes décédés sont pratiquement aussi nombreux pour les migrants que pour les non-migrants (cf. tableau 2). Par ailleurs, I'association mutualiste propose une assurance-décès à ses membres où qu'ils résident : en 2009, sur onze décès survenus aux États-Unis, quatre corps ont été transférés à Tangancícuaro. Les autres familles ont utilisé le pécule versé au Mexique par I'association pour les frais de l'enterrement aux États-Unis (cf. tableau 2), ce qui montre aussi que les envois d'argent (remesas) ne suivent pas uniquement un flux nord-sud mais, à I'occasion, un flux sud-nord.

De telles pratiques dénotent une prise de distance des familles établies aux États-Unis par rapport à la région d'origine de leurs ascendants qui peut s'expliquer par une disparition progressive des migrants fondateurs et de leurs contemporains - sur les vingt décès de 2009, dix ont plus de soixante-quinze ans - et par l'insertion croissante de leurs descendants dans la société étatsunienne. Mon interlocuteur par exemple, membre fondateur de l'association, séjourne avec son épouse au moins trois mois par an en Californie, soit chez la sœur de sa femme, soit chez leur fils. II me confie que l'aîné de leurs petits-enfants vient de passer plus d'un an en Irak comme soldat de l'armée étatsunienne ${ }^{18}$, un signe fort d'appartenance à la société étasunienne.

Malgré ces évolutions, le président et les dirigeants actuels continuent de présenter I'association mutualiste comme un moyen de financer le rapatriement des corps des migrants (la somme versée aux familles est calculée sur cette base) et comme un lieu de sociabilité où les migrants conservent une place privilégiée (l'assemblée annuelle est fixée au mois de décembre, mois traditionnel de retour des migrants, afin de leur permettre $d^{\prime} y$ participer). Son entreprise de pompes funèbres se veut également au service des migrants en proposant notamment une salle pour les veillées funéraires, service perçu comme " étatsunien ", et rare au Mexique où les morts sont veillés à domicile.

16 Interview de Jorge Gudiño Rios, février 2010, gérant de I'association depuis 1995.

17 Interview de Roman Reyes Oropesa.

18 Interview Roman Reyes Oropesa. 
Tableau 2 : Versements pour décès de membres de l'association mutualiste de Tangancícuaro (Tanga) en 2009 (selon date du décès)

\begin{tabular}{|c|c|c|c|c|c|}
\hline Sexe & Âge & Lieu du décès & Lieu de sépulture & $\begin{array}{l}\text { Versement } \\
\text { (en pesos) }\end{array}$ & Observations \\
\hline $\mathrm{H}$ & 69 & Delano, CA, EU & Delano, CA, EU & 65000 & \\
\hline $\mathrm{H}$ & 79 & Tanga, Michoacán, MX & $\begin{array}{l}\text { Tanga, Michoacán, } \\
\text { MX }\end{array}$ & $\begin{array}{l}45415+ \\
\text { services PF }\end{array}$ & $\begin{array}{l}\text { Résidait aux } \\
\text { États-Unis; fut un } \\
\text { des fondateurs }\end{array}$ \\
\hline $\mathrm{F}$ & 76 & Delano, CA, EU & Delano, CA, EU & 65000 & \\
\hline $\mathrm{H}$ & 79 & Tanga, MCH, MX & Tanga, $\mathrm{MCH}, \mathrm{MX}$ & $\begin{array}{l}60120+\text { cercueil } \\
\text { et transport }\end{array}$ & $\begin{array}{l}\text { La veillée a eu } \\
\text { lieu dans la } \\
\text { maison du défunt }\end{array}$ \\
\hline $\mathrm{F}$ & 67 & Tanga, $\mathrm{MCH}, \mathrm{MX}$ & Tanga, $\mathrm{MCH}, \mathrm{MX}$ & $\begin{array}{l}45984+\text { salle et } \\
\text { cercueil }\end{array}$ & \\
\hline $\mathrm{H}$ & 74 & Californie & Tanga, $\mathrm{MCH}, \mathrm{MX}$ & 61375,50 & \\
\hline $\mathrm{H}$ & 42 & Tanga, $\mathrm{MCH}, \mathrm{MX}$ & Tanga, $\mathrm{MCH}, \mathrm{MX}$ & 55812 & \\
\hline $\mathrm{F}$ & $?$ & Phoenix, AR, EU & Tanga, $\mathrm{MCH}, \mathrm{MX}$ & $\begin{array}{l}43657+ \\
\text { cercueil }\end{array}$ & $\begin{array}{l}\text { Nouveau cercueil } \\
\text { au MX }\end{array}$ \\
\hline $\mathrm{H}$ & 77 & Delano, CA, EU & Tanga, $\mathrm{MCH}, \mathrm{MX}$ & 60570 & \\
\hline $\mathrm{H}$ & 86 & Delano, CA, EU & Delano, CA, EU & 65000 & \\
\hline $\mathrm{H}$ & 60 & Tanga, $\mathrm{MCH}, \mathrm{MX}$ & incinéré & $64500+$ salle & \\
\hline $\mathrm{H}$ & 79 & Santa Paula, CA, EU & Tanga, $\mathrm{MCH}, \mathrm{MX}$ & 65000 & \\
\hline $\mathrm{H}$ & 82 & Tanga, $\mathrm{MCH}, \mathrm{MX}$ & Tanga, $\mathrm{MCH}, \mathrm{MX}$ & $\begin{array}{l}55903 \text { + service } \\
\text { PF }\end{array}$ & \\
\hline $\mathrm{H}$ & 57 & $\begin{array}{l}\text { Zapopan, état de } \\
\text { Jalisco, MX }\end{array}$ & Tanga, $\mathrm{MCH}, \mathrm{MX}$ & $26651+$ salle & $\begin{array}{l}\text { Retenue pour } \\
\text { indemnisation de } \\
\text { son vivant }\end{array}$ \\
\hline $\mathrm{H}$ & 67 & Tanga, $\mathrm{MCH}, \mathrm{MX}$ & Tanga, $\mathrm{MCH}, \mathrm{MX}$ & $63771+$ salle & \\
\hline $\mathrm{H}$ & 89 & CA, EU & CA, UE & 64700 & \begin{tabular}{|l|} 
Retenue pour \\
non-cotisation \\
des trois derniers \\
décès
\end{tabular} \\
\hline $\mathrm{H}$ & 84 & $\begin{array}{l}\text { Zamora, Mich, MX (à } \\
15 \text { kms) }\end{array}$ & Tanga, $\mathrm{MCH}, \mathrm{MX}$ & $\begin{array}{l}45079+ \\
\text { services PF }\end{array}$ & \\
\hline $\mathrm{F}$ & 81 & Delano, CA, EU & Delano, CA, EU & 65000 & \\
\hline $\mathrm{H}$ & $?$ & Santa Ana, CA, EU & Santa Ana, CA, EU & & \\
\hline $\mathrm{H}$ & $?$ & Orange, CA, EU & Orange, CA, EU & 30000 pesos & $\begin{array}{l}\text { Versés en dollars } \\
\text { ( } 3 \text { 000 dollars) } \\
\text { aux USA }\end{array}$ \\
\hline
\end{tabular}

Légende $: C A=$ Californie $; E U=$ États-Unis $; M C H=$ Michoacán $; M X=$ Mexique ; $P F=$ service complet de l'entreprise de pompes funèbres de l'association mutualiste 


\section{Un auto-entrepreneur frontalier ${ }^{19}$ (Tijuana, Basse Californie)}

À l'extrême nord du Mexique, dans l'État de Basse-Californie, à Tijuana (1 200000 habitants), ville frontalière dont la population a doublé dans la deuxième moitié du $X X e$ siècle avec l'apport de migrants originaires de tout le Mexique, un bon nombre d'entreprises de pompes funèbres incluent dans leurs services l'envoi des cercueils par voie aérienne dans tout le pays; des transferts uniquement nationaux, mais très fréquents vu la composition de la population locale. La plupart de ces défunts transférés dans d'autres États du Mexique sont décédés dans la ville, certains d'entre eux victimes des luttes violentes et sanglantes entre "familles " mafieuses rivales. Quelques-uns sont amenés par voie terrestre depuis les États-Unis pour être ensuite acheminés dans d'autres régions du Mexique par voie aérienne. À l'inverse, les corps de personnes décédées à Tijuana, la plupart étatsuniennes, souvent des retraités établis en Basse-Californie ou des malades venus se faire soigner dans les hôpitaux de la ville, sont transférés vers le pays voisin.

Si de nombreuses entreprises de pompes funèbres de Tijuana ont pour habitude de réceptionner et d'envoyer des corps par avion, seul Sergio, cinquante ans, fait passer la frontière internationale aux défunts. Il a créé ce service au début des années 1980 à San Diego (Californie) où il travaillait déjà dans une entreprise de pompes funèbres étatsuniennes après avoir été employé très jeune à Tijuana dans le même secteur commercial. L'entreprise de Sergio se réduit à un bureau (un meuble), un fauteuil et une machine à écrire dans l'entrée d'une entreprise de pompes funèbres dont le patron, un ami, I'héberge ; deux téléphones portables qu'il utilise l'un pour le Mexique, l'autre pour les États-Unis ; et une camionnette contenant jusqu'à six cercueils. Sergio, bilingue espagnolanglais, travaille en relation soit avec deux entreprises de pompes funèbres établies de chaque côté de la frontière, en contact l'une avec l'autre, soit directement avec la famille du défunt via une entreprise de pompes funèbres. Son travail consiste à transporter les corps d'une entreprise mexicaine à une entreprise étatsunienne et vice-versa, parfois jusqu'au domicile du défunt si celui-ci se trouve dans le sud de la Californie.

"Beaucoup d'entreprises de pompes funèbres signent un contrat avec nous parce qu'elles nous connaissent depuis longtemps et parce que nous allons chercher le corps à Los Angeles ou bien à la frontière, mais aussi parce que nous obtenons les permis pour faire rentrer le corps au Mexique, nous inscrivons le défunt sur le registre de l'Hôtel de ville, nous obtenons le permis de salubrité et nous mettons le corps dans l'avion, en choisissant un vol qui l'amène directement à l'entreprise de pompes funèbres qui va le recevoir [...] là où il va. À partir de là, l'entreprise de pompes funèbres mexicaine se charge de faire de ce côté-ci [au Mexique] ce que l'entreprise de pompes funèbres étatsunienne a fait de l'autre côté [aux États-Unis] ; elle récupère le corps et elle doit également obtenir un permis pour la sépulture " (Sergio Valencia, Tijuana, avril 2010).

Par la niche commerciale qu'il occupe, Sergio est connu des entreprises de pompes funèbres "locales " (celles de Basse-Californie et des comtés du sud de la Californie ${ }^{20}$ ), ainsi que du consulat mexicain de San Diego, souvent chargé du rapatriement des

\footnotetext{
19 Aux abords de la frontière qui sépare Mexique et États-Unis, on a pour habitude d'utiliser les termes "Ligne " (Línea) pour définir la frontière matérielle et " traverser " (cruzar) pour désigner I'action de passer d'un côté à l'autre. Le terme " traverser " y prend aussi une fonction transitive : on peut "traverser ", au sens de passer, un objet ou un être humain, vivant ou mort, au travers de la Ligne. Parmi ces objets, on peut «traverser » (passer) le corps d'un défunt (cruzar un cuerpo). 20 Par ordre d'importance numérique de la population, Los Angeles (9 519338 habitants), Orange (2 846 289), San Diego (2 813 833), San Bernardino (1 709 434), Riverside (1 545 387), Ventura (753 197) et Impérial (142 361). Source : California Statistical Abstract (2008), [en ligne]. URL : http:// www.dof.ca.gov/HTML/FS_DATA/STAT-ABS/documents/CaliforniaStatisticalAbstract2008.pdf
} 
$\operatorname{corps}^{21}$ qui fait appel à lui pour " rapatrier des citoyens mexicains. II nous contacte pour s'informer sur ce qui revient le moins cher, pour organiser les vols également " (Sergio Valencia, Tijuana, avril 2010), notamment quand il s'agit de transférer les corps dans les États du nord-ouest bordant le Pacifique (Culiacan, Nayarit), relativement proches de la zoneTijuana/San Diego. Contrairement aux entreprises déjà citées, Sergio n'est pas systématiquement en contact avec les familles des défunts mais à l'occasion, dans le cas du transfert d'un défunt étatsunien décédé au Mexique, il aide les membres de la famille à faire les démarches sur place et leur sert de traducteur et de chauffeur.

L'auto-entreprise de pompes funèbres de Sergio représente un chaînon fondamental dans le processus du transfert international par voie terrestre ou pour partie aérienne, puisqu'elle réalise la connexion entre les deux pays, tout en restant le plus souvent en retrait dans l'interaction commerciale et sociale avec les familles des défunts. Le commerce de Sergio est aussi articulé à l'État mexicain, mais il en est nettement moins tributaire que celui de la Purísima, les contrats du consulat ne représentant qu'une partie de son travail.

\section{Une chaîne entrepreneuriale au Mexique et aux États-Unis}

Tous les transferts funéraires internationaux exigent l'intervention d'au moins deux opérateurs, un dans chaque pays. Selon les trajets suivis par les défunts, deux, trois entreprises de pompes funèbres, voire plus, sont nécessaires pour transférer les corps ${ }^{22}$. On a donc à faire à une " chaîne entrepreneuriale " (Péraldi, 1999) de part et d'autre de la frontière.

Dans le cas d'Antonio, décédé en mars 2010, que j'ai suivi d'un bout à l'autre de la chaîne, quatre entreprises de pompes funèbres ont participé au transfert du corps de Los Angeles à Oaxaca, une aux États-Unis et trois au Mexique dont deux frontalières : une entreprise de Los Angeles pour le tronçon par terre de Los Angeles à la frontière ; une entreprise de Tijuana, qui a organisé le transfert de l'autre côté de la frontière puis par avion de Tijuana à Oaxaca ; l'auto-entrepreneur qui s'est chargé du passage de la frontière ; l'entreprise La Purísima à Oaxaca, qui a transporté le cercueil de l'aéroport d'Oaxaca au domicile mexicain du défunt, à San Dionisio Ocotepec (notes de terrain, Oaxaca, mars 2010).

Ces entreprises se sont partagé le travail. Une chaîne commerciale s'est mise en place, en partie en dehors des familles et en collaboration avec les États. Les familles n'ont eu de contacts qu'avec les entreprises de Los Angeles pour ceux des membres vivant aux États-Unis au moment du départ du défunt et celle de San Dioniso Ocotepec pour les membres mexicains de la famille, au moment de la remise du corps au domicile mexicain ${ }^{23}$. L'entreprise de Tijuana et l'auto-entrepreneur Sergio n'ont eu de relations qu'avec les deux autres entreprises impliquées dans le transfert. De plus, tous ont été en contact avec plusieurs niveaux étatiques nationaux (Mexique et États-Unis) et régionaux

\footnotetext{
21 Le consulat est amené soit à organiser et payer seul le transfert des migrants décédés en passant la frontière illégalement, soit à aider les familles des autres défunts mexicains à organiser ce transfert (Lestage, 2008 et 2010).

22 Je n'ai pas mentionné le transfert des urnes funéraires parce que les cendres voyagent la plupart du temps sans passer par les entreprises de pompes funèbres. Même si cette pratique est interdite, comme le signale la vice-consul mexicaine à San Diego (interview avril 2010).

23 Par ailleurs, le contact de la famille au Mexique avec I'entreprise La Purísima a été bref, le temps de décharger le cercueil, de confier les documents nécessaires à l'enterrement aux parents du mort et de leur faire signer le document de remise du corps.
} 
(Oaxaca, Californie) via des administrations chargées des contrôles, et dans le cas de l'État régional mexicain du Oaxaca parce qu'il a suivi, confié et payé le dernier tronçon du voyage à l'entreprise La Purísima.

Notons que les entreprises qui composent la chaîne commerciale ne sont pas dirigées par des " entrepreneurs transnationaux " au sens de Portes et al. (2002), à savoir " des immigrants dont les activités commerciales exigent de fréquents voyages à l'étranger et qui dépendent de leurs contacts dans le pays d'origine pour le succès de leur entreprise " (Portes, Guarnizo, Haller, 2002 : 287 ; ma traduction) puisque leurs directeurs, gérants ou propriétaires résident dans le pays où ils travaillent, leur pays d'origine dans la très grande majorité des cas. Ce ne sont pas non plus des "entreprises de reproduction identitaire " (Ma Mung et Guillon, 1992) qui mettraient en scène le déplacement du corps, car elles offrent un service funéraire discret dans le pays d'origine des migrants. Aux États-Unis, certaines peuvent être qualifiées de "commerces ethniques ", leurs propriétaires utilisant l'origine nationale et une spécificité socio-culturelle qui y serait attachée (Ma Mung, 1992 ; Raulin, 2000) pour attirer des clients (Lestage, 2008a). Mais ce n'est pas le cas au Mexique où ces entreprises ne présentent aucun signe distinctif ; la plupart souhaitant attirer aussi une clientèle qui n'est pas concernée par les transferts funéraires internationaux. Enfin, les entreprises de pompes funèbres mexicaines de cette chaine commerciale $n^{\prime}$ ont pas toutes été créées par des migrants ou avec l'argent envoyé par les migrants comme Massey et Parrado (1998) I'ont montré pour d'autres commerces, en soulignant que les envois d'argent des migrants ne servent pas uniquement à la consommation quotidienne, mais peuvent conduire au soutien ou à la création d'entreprises par ces mêmes migrants ou par leur entourage au Mexique ${ }^{24}$. En définitive, les entreprises de pompes funèbres spécialisées dans les transferts funéraires internationaux ne sont impliquées dans le processus migratoire qu'à travers leur clientèle et les services et produits proposés.

II s'agit d'une clientèle variée et chaque type de client a des attentes spécifiques : les États régionaux s'attachent au transfert régional du cadavre, les consulats aux migrants décédés au passage de la frontière, etc. Au Mexique, I'offre est peu diversifiée et la plupart des entreprises de pompes funèbres proposent des services et des produits identiques. En revanche, aux États-Unis, les familles de migrants ou de descendants de migrants mexicains ont davantage de choix : elles peuvent s'adresser à des entreprises de pompes funèbres en fonction de critères économiques ou géographiques, ou bien de critères " ethniques ". Entendons par là des entreprises de pompes funèbres spécialisées dans les transferts vers le Mexique qui vendent des services funéraires perçus comme " mexicains ": dont le nom (Guadalupana, Aztlan dans le comté de San Diego par exemple) ou la décoration (une maison de style mexicain, des meubles et des symboles mexicains ${ }^{25}$ ) évoquent le Mexique (Lestage, 2008a).

Quelle que soit l'image que donnent les entreprises étatsuniennes de pompes funèbres ethniques destinées aux Mexicains et Mexico-américains, leurs pratiques sont

\footnotetext{
24 Des chercheurs l'ont démontré pour d'autres migrants aux États-Unis : les Dominicains (Portes et Guarnizo, 1990) ; les Salvadoriens (Landolt et al., 1999), etc.

25 "Le nom, mais aussi l'aspect de l'entreprise de pompes funèbres, mettent en scène des éléments de la mexicanité. L'entreprise Aztlan est une petite maison blanche avec une cour remplie de pots de fleurs et de bougainvillées. À l'intérieur, elle a tout d'une maison mexicaine traditionnelle, des dalles ocre-rouge du sol, aux meubles en osier et cuir, des ornements comme l'arbre de la vie à la reproduction d'un tableau de Diego Rivera que I'on relie tous au Mexique. De plus, les employés sont totalement bilingues espagnol-anglais, et l'entreprise est située dans une zone résidentielle [de San Diego] où résident une proportion élevée de Mexicains et de Mexicainsaméricains " (Lestage, 2008a : 218).
} 
identiques à celles des entreprises étatsuniennes non-ethniques et leurs services pensés pour une clientèle imprégnée de représentations plus étatsuniennes que mexicaines, comme on le constate au premier regard en observant leurs locaux. En effet, une des différences notables entre les entreprises des deux pays est le degré de visibilité ou d'invisibilité de la mort et de ses attributs dans les boutiques et/ou bureaux accueillant les clients : au Mexique, des cercueils grandeur nature sont exposés dans le magasin et sa vitrine, alors qu'aux États-Unis (comme en France du reste) tout est discret et suggéré, car la mort est un " tabou marchand " et ne s'expose pas (Trompette et Boissin, 2000 ; Trompette, 2008). D'autres différences de comportement des familles vis-à-vis de la mort et des défunts sont à noter, en particulier, comme on l'a déjà indiqué, la pratique de la veillée à domicile dominante au Mexique, alors que les morts sont gardés dans les chambres funéraires aux États-Unis. Ajoutons à cela des différences au Mexique même, où les pratiques et croyances entourant le décès et le traitement du défunt varient entre ruraux et urbains ainsi qu'entre indiens (relevant de groupes ethniques aux pratiques et croyances spécifiques) et non-indiens ${ }^{26}$, variations observables également à l'occasion du retour des cadavres des migrants dans leur lieu d'origine ${ }^{27}$.

Dans de nombreux cas de mort en migration et de transfert de corps, les rites funéraires sont dédoublés (Lestage, 2008b et 2009). Les entrepreneurs de pompes funèbres interviewés remarquent qu'il est fréquent que la famille au Mexique achète un nouveau cercueil pour une nouvelle série de rituels : veillée familiale, messe. Les deux messes d'enterrement (aux États-Unis puis au Mexique), tout comme les deux veillées et les deux cercueils du mort, sont, de mon point de vue, des éléments de contrôle du cadavre que chaque partie de la famille, dans chaque pays, souhaite exercer sur le défunt. En effet, " la nécessité d'établir un contrôle sur le cadavre s'exprime selon des modalités et des degrés différents" (Saravia, $1993: 8$ ) chez les entrepreneurs de pompes funèbres (comme I'a montré Saravia en comparant funeral directors américains et fossoyeurs portugais ; Saravia, ibid.) et dans les familles des défunts.

Selon le lieu de résidence et la durée d'installation du défunt et de ses proches, les pratiques relatives au traitement de la mort évoluent, se métissent, ou sont transposées d'un pays à l'autre. On a vu que l'entreprise La Purísima loue un " kit veillée " aux familles qui souhaitent veiller le mort dans leur maison, une pratique majoritaire dans l'État du Oaxaca qui ne semble pas avoir été modifiée par les migrations. On a vu également que I'entreprise dépendant de l'association mutualiste met à disposition des familles une salle pour les veillées funéraires sur le modèle des salles funéraires étatsuniennes. Alors que le gérant remarque que cette salle est peu utilisée, les statistiques montrent qu'en 2009, sur les vingt décès pris en charge par l'association, près d'une famille sur deux a utilisé des services de l'entreprise (achat du cercueil, location du corbillard) et une sur quatre a utilisé la salle funéraire. Sur ces quatre familles, une seule veillait un migrant, fondateur de l'association. Une telle situation laisse penser que de nouvelles pratiques se mettent progressivement en place, du moins localement, puisque ce sont en majorité les familles

26 Ces différences sont également marquées lors du rituel annuel du Jour des Morts, les indiens croyant au retour provisoire de leurs défunts parmi eux à ce moment-là, les autres jouant avec l'idée de la mort (en famille et entre amis, mais aussi dans les médias, jeu qui entretient un commerce ludique d'objets liés à la mort).

27 Par exemple, le groupe de parenté qui se charge du défunt diffère, car une telle responsabilité est liée à la forme d'union matrimoniale et d'alliance - dans certains groupes sociaux, si une femme décède, ce sera le groupe de son mari qui s'occupera du traitement du corps (Lestage, 2009) - et à d'autres règles locales qui établissent quels seront les porteurs du corps, les modalités de la période de deuil qui suit, etc. 
des non-migrants qui utilisent la salle pour veiller les morts ${ }^{28}$.

En offrant des services "traditionnels" (le kit veillée) ou "nouveaux" (la salle pour veillée) qui répondent à des demandes réelles ou supposées de leurs clientèles, les entreprises de pompes funèbres spécialisées dans le transfert des défunts jouent un rôle dans le développement de pratiques funéraires perçues comme mexicaines ou étatsuniennes et dans l'évolution des conditions de la réappropriation du corps par les familles des migrants au Mexique. Cette "chaîne entrepreneuriale " implique les entreprises mexicaines et étatsuniennes qui commercialisent les transferts des défunts et constituent un marché de services (transport des cercueils, gestion administrative des voyages) et de produits (vente de cercueils et autres objets funéraires, vente d'assurance-décès) structuré et organisé à travers les deux pays.

\section{Une chaîne bi- et trans-nationale ?}

Cette " chaîne " n'est pas uniquement entrepreneuriale, car elle articule aux processus commerciaux des processus sociaux et politiques. Elle inclut les familles des défunts à l'origine de ces flux (Lestage, 2008b et 2009), les associations de migrants ou les églises qui participent au financement des obsèques et à l'accompagnement des familles, et les États fédéraux et fédérés mexicains et étatsuniens, régulateurs des flux de restes humains entre les deux pays (Lestage, 2008a ; Felix, 2011). Soulignons aussi que cette chaîne a pour toile de fond des représentations sociales qui lient la mort à la nation mexicaine, faisant d'elle le " totem " de cette nation comme l'a montré Lomnitz (2006), représentations que I'on retrouve dans les chansons populaires (Felix, 2011), dans les discours des individus et dans ceux des gouvernants (Lomnitz, 2006).

\section{Les entreprises de pompes funèbres, les États-nations et le contrôle des défunts}

Les États nationaux et régionaux imposent un cadre administratif au transfert des défunts afin de les contrôler et, éventuellement, de les utiliser dans la rhétorique régionale ou nationale. Ce commerce est donc encadré par des législations nationales et internationales strictes résultant d'accords multilatéraux soit européens (Berlin, 10 février 1937 ; Strasbourg, 26 octobre 1973 ${ }^{29}$ ) soit américains (7 octobre 1966, Pan American World Health Organization ${ }^{30}$ ) qui présentent quelques divergences, les législations européennes étant préoccupées par le cercueil alors que les législations étatsuniennes le sont par le corps et sa conservation (Chaïb, $2000: 49$ ).

Chacun des États-nations gère la partie du processus qui le concerne en s'appuyant sur différentes administrations, notamment sanitaires, les préoccupations hygiéniques étant mises en avant dans toutes les législations. Dans les procédures, chacun respecte les prérogatives et les territoires nationaux, car, du point de vue des États, l'administration

\footnotetext{
$28 \mathrm{Ce}$ n'est pas le propos de cet article, mais on peut se demander pourquoi les familles des migrants défunts n'ont utilisé la salle qu'exceptionnellement. S'agit-il, là encore, d'exercer un contrôle différent sur le défunt, la veillée dans l'entreprise de pompes funèbres étant assimilée à une pratique étatsunienne ? Ou ce lieu est-il perçu comme trop impersonnel pour se réapproprier le corps du migrant, qui s'est éloigné de sa famille de son vivant, géographiquement du moins, et qui requiert une ambiance d'autant plus intime et familière ?

29 Voir http://www.afif.asso.fr/francais/conseils/legislation/sommaireloi.html (consulté le 3 juin 2012).

30 XVII Pan American Sanitary Conference, XVIII Regional Committee Meeting, Resolution XXIX. Voir http://www.state.gov/documents/organization/86578.pdf
} 
de la mort est " une des pierres angulaires de leur souveraineté " (Lomnitz, $2006: 55)^{31}$. Une souveraineté que l'État mexicain manifeste quand les décès des migrants mexicains impliquent celle de l'État étatsunien, soit parce que la mort est liée au passage illégal de la frontière, rendu extrêmement dangereux par les politiques de fermeture mises en place par les États-Unis depuis 1994 et davantage depuis 2001, soit parce qu'il s'agit d'une condamnation à mort aux États-Unis, soit encore parce qu'un fonctionnaire est mis en cause $^{32}$. Bien que les différents niveaux de l'État mexicain interviennent dans les transferts des morts mexicains en aidant les familles à les financer et à les organiser, relevant d'un dispositif de " gestion politique de la souffrance " (Lestage, 2010) ${ }^{33}$, les transferts de corps se font généralement sans bruit médiatique ni politique.

Le passage d'un pays à l'autre suppose l'obtention de plusieurs certificats administratifs et requiert des autorisations de transport délivrées par les administrations concernées. Hormis ces contraintes, le choix des entreprises de pompes funèbres est, en principe, libre. Or, on a vu que certaines d'entre elles ont tout intérêt à mobiliser leurs réseaux au sein des gouvernements en place ou dans les consulats pour avoir des clients. En effet, selon Trompette, le choix d'une entreprise de pompes funèbres se fait peu en fonction du jugement d'un client actif qui choisirait " en toute raison après comparaison " (Trompette, 2004.). Le client, rendu passif par la dimension ontologique et la rareté de l'évènement, est " capté " par l'entreprise de pompes funèbres, généralement à partir des hôpitaux et des chambres funéraires (Trompette, 2008). Dans le cas du transfert funéraire international, on a souligné que cette captation mobilise d'autres circuits : les membres d'une association de migrants, un réseau transfrontalier d'entreprises de pompes funèbres, la connaissance de fonctionnaires ayant des positions privilégiées dans la circulation des défunts (principalement dans les consulats mexicains aux États-Unis qui enregistrent les décès et en financent une partie, et dans les États régionaux mexicains qui financent la partie locale du transfert).

Nos entreprises représentent trois cas de figure qui vont d'une dépendance quasi totale vis-à-vis de l'État (celle de l'entreprise familiale qui s'est spécialisée progressivement dans les transferts pour le compte de l'État régional du Oaxaca) à l'autonomie partielle de l'entrepreneur solitaire qui collabore parfois avec l'État mexicain par l'entremise du consulat de San Diego, jusqu'à l'autonomie totale de l'association mutualiste. L'indépendance de cette dernière peut s'expliquer par son implantation durable dans la localité et par la confiance de ses habitants. Une telle autonomie est d'autant plus remarquable que l'État régional du Michoacán apporte son aide au rapatriement des défunts depuis le début des années 2000. C'est aussi le plus avancé de tout le Mexique dans

\footnotetext{
31 Comme le souligne aussi Foucault (2004) sur le rapport de l'État au corps des citoyens et les travaux s'inspirant de ses écrits.

32 Ce fut le cas en avril 2010, à la suite de l'assassinat à New York d'un migrant indien mexicain ivre par un policier étatsunien. Les autorités mexicaines réagirent alors en mobilisant des représentants de la nation mexicaine autour du cadavre. Le consul à New York s'occupa personnellement de l'affaire, les autorités régionales de l'État d'origine du défunt (Oaxaca) accueillirent le corps à I'aéroport et certaines I'accompagnèrent jusqu'au fin fond de la campagne mexicaine sous I'œil attentif des médias mexicains informés de l'évènement et prêts à le diffuser (Source : Décès de Marcos de Jesus Alvarez, survenu à quarante-sept ans, appartenant au groupe triqui, unilingue triqui, de la localité de Santa CruzTilapa, district de Juxtlahuaca, état du Oaxaca (information obtenue auprès de Araceli Lopez, responsable du service à I'Institut Oaxaqueno d'Aide au Migrant (IOAM). Article dans le journal EITiempo, 25 mars 2010 ; reportage de la chaîne de télé UNIVISION). 33 Dispositif qui ne concerne pas seulement l'aide au transfert des corps, mais aussi celle en rapport avec une prise en charge de souffrance (aide aux prisonniers mexicains, pour obtenir des visas dits humanitaires, etc.).
} 
I'aide aux migrants puisqu'il est le seul à avoir un Secrétariat d'état d'aide aux migrants ${ }^{34}$ (Lestage, 2010).

\section{Les effets d'un « mode de vie transnational »}

Les entreprises de pompes funèbres s'inscrivent également dans un espace social " transnational ", au sens donné par Glick Schiller et Lewitt " d'un ensemble de réseaux entrelacés de relations sociales, à travers lequel les idées, les pratiques et les ressources sont inégalement échangées, organisées et transformées ${ }^{35}$ " (Glick Schiller et Lewitt, 2004 : 9). Ce vaste tissu de liens sociaux entraîne un " mode de vie transnational " (transnational living) (Guarnizo, 2003) duquel participent les transferts internationaux de cadavres qui mobilisent des réseaux économiques, familiaux, associatifs et politiques de part et d'autre de la frontière. Dans ce " mode de vie transnational ", deux processus sont en jeu, selon Guarnizo (2003) : la reproduction de pratiques socio-culturelles en pays d'immigration et le maintien d'échanges destinés à entretenir des relations sociales, politiques et économiques entre pays d'origine et pays de résidence. À mon sens, il convient d'en ajouter au moins un troisième : la transposition et l'aménagement de pratiques socio-culturelles du pays d'immigration vers le pays d'émigration - comme on l'a constaté notamment avec la pratique naissante de la chambre funéraire payante mise à disposition par l'entreprise de pompes funèbres. Ces processus entraînent des flux de biens d'un pays à l'autre qui suscitent ou construisent des marchés, notamment ceux de la communication, du transport et de l'immobilier (Guarnizo, 2003).

J'ajouterai à ces marchés celui du transfert des restes humains qui relève, à mon sens, de ces processus : il permet d'entretenir des relations avec la parentèle émigrée ou restée au pays; il renvoie au désir des familles de s'inscrire dans un groupe généalogique territorialisé, que celui-ci situe son fondement dans le pays d'origine ou dans celui d'accueil ; il entraîne la transposition de pratiques socio-culturelles et commerciales dans les lieux d'émigration ainsi que la reproduction de pratiques socio-culturelles dans les lieux d'immigration. Sur le plan symbolique, le partage de l'émotion de la perte du défunt, les tensions et les conflits relatifs à l'emplacement de la sépulture renforcent les liens. Ils réactivent aussi le sens des origines et l'attachement aux lieux. Le marché des transferts des restes des migrants est donc exemplaire d'un " mode de vie transnational ". Par ailleurs, il s'est constitué en niche économique, occupée par des acteurs migrants (I'entreprise ethnique située à San Diego) ou non-migrants (les trois entreprises étudiées) qui " capturent " (Guarnizo, 2003 : 680) ainsi cette " chaîne entrepreneuriale de la mort ".

\section{Conclusion}

Dans son ouvrage sur les migrants tunisiens défunts, Chaïb (2000) cite le passage où Victor Hugo raconte le transfert, par le train, du corps de sa femme, tout en soulignant que ce " rapatriement de la dépouille mortelle fait partie du rite funéraire " (Chaïb, 2000 : 55-56). Notre interlocuteur mexicain, Roman, qui voyagea en train avec le cercueil de son frère de la Californie au Michoacán durant une semaine, ne voit pas ce transfert comme un rite funéraire. Alors que Victor Hugo rend visite à sa défunte femme dans un wagon

\footnotetext{
34 Au Mexique, depuis la fin des années 1990, chaque État fédéré a créé une agence d'aide aux migrants. Apparemment identiques, ces agences ont cependant des noms différents ("Secrétariat d'État " au Michoacán, "Institut d'Aide au Migrant " dans l'État du Oaxaca) et sont impliquées de façon très variable dans l'administration et le financement de questions liées à la migration (pour plus de précisions voir Yrizar et Alarcon, 2010 ; Lestage, 2010).

35 " As a set of multiple interlocking networks of social relationships through which ideas, practices and resources are unequally exchanged, organized and transformed ".
} 
transformé en chapelle, Roman accompagne son frère "comme un bagage [dit-il] ". Le " cercueil-bagage " qu'il nous décrit est plus proche du " corps-marchandise conditionné sous une lettre de transport " dont parle Chaïb (2000:50) que du rituel. Car le cadavre constitue aujourd'hui un objet " marchandisé ", "classé, répertorié, trié, codifié " (Chaïb, ibid.) dont le déplacement est pris en charge par le commerce du transfert funéraire international. Ce commerce, qui répond à une demande de biens et de services engendrée par le " mode de vie transnational ", peut donc être analysé, très justement, comme un effet de la migration des Mexicains aux États-Unis.

Mais il n'en est pas seulement un effet. II I'alimente à son tour en produisant ses propres flux - de morts, d'argent, d'échanges entre entreprises, entre familles et entre États. Et il fait des entreprises de pompes funèbres impliquées dans ce marché, des relais : relais vis-à-vis des familles des défunts, car l'entrepreneur est souvent en relation, à la fois, avec la famille aux États-Unis et au Mexique ; relais des États puisque ces transferts constituent un enjeu pour les gouvernements et sont des signes politiques; relais d'autres entreprises, mexicaines ou étatsuniennes, soit parce qu'elles manquent de savoir-faire dans ce domaine et recourent à des collègues spécialisés, soit parce que chaque entreprise ne travaille que sur le territoire national et nécessite un correspondant dans le pays voisin ; relais d'associations de migrants et de non-migrants, puisque ces transferts sont parfois pris en charge par des collectifs plus ou moins organisés qui ont à faire aux entrepreneurs.

Il s'agit bien de relais, mais pas de passeurs ou de médiateurs qui guideraient les familles en deuil et les assisteraient, comme le laisse entendre Trompette pour les entreprises de pompes funèbres en France (2008), un rôle que jouent localement, au Mexique et aux États-Unis, des leaders familiaux, sociaux, religieux ou politiques. Même si les familles ont tendance à attribuer la responsabilité de l'état du corps du défunt à celui qui l'apporte (Lestage, 2008b), que ce soit positivement et avec gratitude (quand le défunt arrive dans un beau cercueil et en bon état) ou négativement et avec des injures (quand le défunt est nu dans le cercueil par exemple ${ }^{36}$ ), les entrepreneurs de pompes funèbres sont des relais au sens de guides techniques, qui possèdent des compétences permettant à leurs clients et interlocuteurs de rapatrier les défunts dans les meilleures conditions possibles en réduisant la durée, le coût et la complexité administrative du transfert et en ménageant la souffrance des familles.

Pour les entrepreneurs des transferts internationaux des morts, ce commerce est une activité économique productrice de richesse qui repose sur un processus migratoire établi et pérenne ayant façonné un ensemble de liens familiaux, régionaux et nationaux entretenus par-delà les frontières par des migrants, des non-migrants, des fonctionnaires, des politiques et des entrepreneurs. Tous ces acteurs participent au fonctionnement de cette chaîne économique, articulée au social et au politique, qui éclaire les interactions complexes que produit le fait migratoire et donne raison à Simon pour qui la migration est "l'une des formes de la respiration et de la communication des sociétés " (Simon, 1995).

36 Notes de terrain, Oaxaca, 21 avril 2008. 


\section{Références bibliographiques}

Bernard Julien (2008) Bonne distance et empathie dans le travail émotionnel des pompes funèbres. L'analyse des interactions en milieu professionnel, Journal des anthropologues, 114-115, pp. 109-128.

Felix Adrian (2011) Posthumous transnationalism: Postmortem Repatriation from the United States to Mexico, LARR, 46 (3), pp. 157-179.

Foucault Michel (2004) Naissance de la biopolitique, Cours au collège de France 19781979, Hautes Études, Paris, Gallimard-Seuil, 356 p.

Glick Schiller Nina, Basch Linda and Szanton Blanc Cristina (1995) From Immigrant to Transmigrant: Theorizing Transnational Migration, Anthropological Quarterly, 68 (1), pp. 48-63.

Glick Schiller Nina and Lewitt Peggy (2004) Conceptualizing Simultaneity. A Transnational Social Field Perspective On Society, International Migration Review, 38 (3), pp. 1002-1039.

Guarnizo Luis (2003) The Economics of Transnational Living, International Migration Review, 37 (3), pp. 666-699.

INEGI (2010) Censo de población y vivienda, [en ligne] consulté le 03/06/2012. URL : http:// www3.inegi.org.mx/sistemas/iter/consultar_info.aspx

Lestage Françoise (2010) The political management of suffering. New practices by the Mexican state(s) with their emigrants, communication aux Journées Mobilities, Regulations and Citizenship, Université de Chicago et Paris Diderot.

Lestage Françoise (2009) Vivants et morts dans les migrations mexicaines : un système de relations inscrit dans la mobilité, in Laurent Faret, Geneviève Cortes, Virginie Baby-Collin et Hélène Guétat Éds, Migrants des Suds. Acteurs et trajectoires de la mobilité internationale, IRD, Université de Montpellier, Presses Universitaires du Mirail (PUM), pp. 431-452.

Lestage Françoise (2008a) Apuntes relativos a la repatriación de los cuerpos de los mexicanos fallecidos en Estados Unidos, Migraciones Internacionales, 4 (4), pp. 217-228, [en ligne] consulté le 03/06/2012. URL : http://www2.colef.mx/migracionesinternacionales/ revistas/MI15/n15-209-220.pdf

Lestage Françoise (2008b) Le dernier voyage des migrants mexicains. Ethnographie du retour des défunts, Caravelle, 91, pp. 131-148

Ma Mung Emmanuel (1992) L'expansion du commerce ethnique : Asiatique et Maghrébins dans la région parisienne, Revue Européenne des Migrations Internationales, 8 (1), pp. 39-59.

Ma Mung Emmanuel et Guillon Michelle (1992) Dispositif économique et ressources spatiales : éléments d'une économie de diaspora, Revue Européenne des Migrations Internationales, 8 (3), pp. 175-193.

Massey Douglas and Parrado Emilio (1998) International Migration and Business Formation in Mexico, Social Science Quaterly, 7 (1), pp. 1-20. 
Peraldi Michel (1999) Marseille : réseaux migrants transfrontaliers, place marchande et économie de bazar, Cultures \& Conflits, [en ligne], consulté le 10/03/2012. URL : http:// conflits.revues.org/index232.html

Portes Alejandro (1997) Globalization from Below: The Rise of Transnational Communities, Working Paper du Center for Migration and Development, Princeton University, 27 p.

Saravia Clara (1993) Le mort maquillé. Funeral directors américains et fossoyeurs portugais, Terrain, 20, [en ligne]. URL : http://terrain.revues.org/3061

Simon Gildas (1995) Géodynamiques des migrations internationales dans le monde, Paris, PUF, $429 \mathrm{p}$.

Trompette Pascale (2008) Le marché des défunts, Paris, Presses de Sciences Po, 300 p.

Trompette Pascale et Boissin Olivier (2000), Entre les vivants et les morts : les pompes funèbres aux portes du marché, Sociologie du travail, 42 (3), pp. 483-504.

Trompette Pascale et Caroli Sandrine (2004) En aparté avec les morts... Peurs, larmes et rire au travail : les métiers du funéraire, Terrain, 43, pp.63-84.

Yrizar Barbosa Guillermo and Alarcon Rafaël (2010) Emigration Policy and State Governments in Mexico, Migraciones Internacionales, 5 (4), pp. 165-198. 


\section{Françoise Lestage}

\section{$\cdots$ Entre Mexique et États-Unis : la chaîne entrepreneuriale de la mort des migrants}

Cet article se propose d'interroger la place qu'occupe le commerce du transfert funéraire international dans le processus migratoire en se fondant sur l'ethnographie de trois entreprises mexicaines de pompes funèbres, situées dans trois régions distinctes, et spécialisées dans le transfert des corps de ou vers les États-Unis. II montre comment les entreprises de pompes funèbres mexicaines qui commercialisent les transferts des restes humains forment, avec leurs homologues aux États-Unis, un marché binational de services et de produits organisé comme une " chaîne entrepreneuriale de la mort " pour laquelle le passage d'un pays à l'autre constitue le fonds de commerce. Il souligne aussi comment les rapports de ces entreprises avec les migrants, leurs familles et les États varient selon la localisation géographique, la temporalité et les fluctuations des politiques régionales et nationales mexicaines envers leurs émigrants. II en conclut que ces entreprises sont autant des effets que des relais de la migration mexicaine aux États-Unis et de l'espace social transnational qu'elle produit.

\section{Between Mexico and the United States: The Entrepreneurial Chain of Migrants' Death}

This article aims to examine the place of the funeral industry transfer in the international migration process. It is based on the ethnography of three Mexican funeral homes, located in three distinct regions, and specialized in transferring human remains from, or to, the United States. It shows how funeral companies that do this business in Mexico form, with their counterparts in the United States, a bi-national market of services and products, organized as an "entrepreneurial chain of death" for which business is the transition from one country to another. It also highlights how the relations of these companies with the migrants, their families and states vary according to geographical location, temporality, and fluctuations in regional and national policies towards the Mexican emigrants. He concluded that these companies are effects and relays of Mexican migration to the United States and of the transnational social space it produces.

\section{Entre México y Estados-Unidos: la cadena empresarial de la muerte de los migrantes}

Este artículo pretende cuestionar el lugar que ocupa la industria de los traslados funerarios internacionales en el proceso migratorio, basándose en la etnografía de tres funerarias mexicanas, localizadas en regiones distintas, y especializadas en el traslado de cuerpos humanos desde o hacia los Estados-Unidos. Muestra como las funerarias mexicanas que comercializan los traslados de restos humanos forman con sus homologas estadounidenses, un mercado binacional de servicios y productos, organizado como una "cadena empresarial de la muerte» para la cual el fondo de comercio es el pasar de un país a otro. Recalca también como las relaciones que mantienen las funerarias con los migrantes, con sus familias, y con el Estado, se diferencian según la localización geográfica, la temporalidad, y las fluctuaciones de las políticas regionales y nacionales mexicanas para con sus emigrantes. Concluye que estas funerarias son tanto los efectos como los relevos de la migración mexicana a Estados Unidos, y del espacio social transnacional que ella produce. 